\title{
Le mystère de l'homogénéité génétique de l'humanité
}

Quand les scientifiques comparent les séquences d'ADN de deux êtres humains habitant sur des continents différents, elles apparaissent bien plus semblables entre elles que ne le sont deux séquences d'ADN appartenant à deux gorilles de la même forêt de l'Est africain. En un mot, notre espèce présente une similitude génétique bien plus importante que nos proches apparentés, les singes antropomorphes. Cela reste un mystère, bien qu'il existe une amorce d'explication: si nos ancêtres étaient passés par un "goulot d'étranglement", avec une diminution brusque du nombre de la population sur une courte période, la population survivante émergeant du goulot aurait perdu énormément de sa variabilité génétique. Plusieurs équipes d'anthropologistes, de généticiens et de biologistes des populations ont récemment proposé que l'homme moderne serait, en fait, issu d'une population d'à peu près 100000 individus qui se serait réduite, à un certain moment au cours des dernières 400000 années, à 10000 femmes et hommes capables de se reproduire. Ce groupe d'individus est ce que les biologistes appellent une population "effective" (la population totale, incluant les individus plus âgés et les enfants, a dû être plus importante) [1].

\section{Quelques chiffres}

Naoyuki Takahata, un généticien des populations japonais, a utilisé la "théorie de la coalescence", pour remonter le temps et aboutir à ce chiffre de 10000 à partir des gènes contemporains de 5 milliards d'êtres humains [2]. Pour cela, il faut trou- ver d'abord combien il y a de versions différentes d'un gène spécifique humain moderne (allèles). Il faut ensuite connaître la rapidité à laquelle les nucléotides mutent à cet endroit. Cette valeur est obtenue en comparant les gènes entre différentes espèces de singes et l'homme, la divergence entre ces espèces étant connue et basée sur les données des fossiles. Prenons l'exemple simple de la séquence nucléotidique de deux allèles pour le même fragment d'ADN. Le nombre de différences de nucléotides entre les deux allèles, divisé par le nombre total de nucléotides, mesure la distance génétique entre les deux allèles. Sachant combien de temps il a été nécessaire à ces mutations pour s'accumuler, on peut alors déduire quand les deux allèles coalescent en un ancêtre commun. Ces valeurs sont ensuite insérées dans une équation qui calcule la taille effective de la population de l'espèce pour ce gène : elle est égale à la distance génétique divisée par quatre fois le taux de mutation [2]. Ainsi, plus le degré de diversité entre allèles est important, plus l'âge de leur ancêtre commun est ancien et plus la population nécessaire à maintenir cette diversité à travers le temps est importante. Toutefois, regarder un gène n'est pas suffisant et pour atteindre un seuil statistique significatif, il faut en étudier une cinquantaine. Takahata et al. ont donc étudié 50 gènes neutres*. La distance génétique moyenne pour les 50 gènes est de 0,0008 substitution par site nucléotidique. Le taux estimé de mutations est de 1 substitution tous les 50 millions de générations $\left(2.10^{-8}\right.$ substitu- tions par site par génération). Lorsque ces deux valeurs sont rentrées dans l'équation, la taille de la population effective pour ces 50 gènes est de 10000 . Les auteurs ont donc conclu que la taille de la population effective était aux alentours de 10000 individus qui auraient vécu quelque temps après que notre espèce soit apparue il y a 400000 ans et avant l'explosion démographique de la population humaine d'il y a 10000 ans [3].

Mais la question restait entière de savoir si ce groupe était relativement stable ou s'il résultait d'une population plus ancienne plus importante. Takahata a alors étudié des portions plus diverses du génome: les gènes du complexe majeur d'histocompatibilité (gène $H L A$ ). En effet, la diversité de certaines allèles HLA est le reflet d'une existence très ancienne. Par exemple, certains allèles humains $D R B 1$ sont partagés avec les grands singes et autres primates qui ont divergé de l'homme il y a 6 millions d'années. Ces allèles sont donc plus anciens que notre espèce. Après avoir ajouté les effets de la sélection naturelle sur les allèles $H L A$ dans les calculs de coalescence, les chercheurs ont conclu que pour maintenir une telle diversité, si longtemps, les premiers ancêtres humains devaient avoir une taille de population de 100000 pendant les quelques millions d'années avant l'émergence de l'homme moderne.

\footnotetext{
* Un gène neutre est défini comme un gène non affecté par la sélection naturelle car il ne confere ni avantage ni desavantage individuel ou reproductif.
} 
Mais il fallait également savoir si les gènes $H L A$ avaient subi un goulot d'étranglement. Takahata et al. ont donc utilisé une simulation prenant en compte l'ensemble des allèles $D R B 1$ dans la population actuelle, et ont calculé la taille de population minimale requise pour que ces allèles aient été présents chez les générations qui vivaient il $y$ a 400000 ans (quand le premier Homo sapiens est apparu). La réponse a été 10000 , ce qui a amené Takahata à conclure qu'avant l'apparition de l'homme moderne, la taille de la population fluctuait aux alentours de 100000 individus. Entre 400000 et 800000 ans, elle a chuté aux environs de 10000 individus et serait restée proche de ce nombre jusqu'à il y a 12000 ans [1].

Enfin, il y a quelques années, les anthropologistes Henry Harpending et Alan Rodgers de l'université de Pennsylvanie [3] ainsi que John Maynard Smith dans le Sussex en Angleterre [4] avaient déjà évoqué ce chiffre de 10000 sur des données fondées sur l'étude de l'ADN mitochondrial (ADNmt).

Ainsi donc, par trois approches différentes (gènes neutres, gènes $H L A$, ADNmt), le même nombre de 10000 individus est avancé pour la taille de la population humaine à son origine.

Mais que veulent dire ces chiffres? Pour Takahata et Klein [2], le passage de 100000 à 10000 individus indique clairement qu'il y a eu un goulot d'étranglement et que ce goulot est responsable de la perte de diversité génétique. En revanche, pour Francisco Ayala, un autre généticien des populations de l'université de Californie, ce chiffre de 10000 n'est pas un goulot. Dix mille est en effet le nombre de la population effective de nombreuses espèces, des macaques aux phoques, et ces espèces ont un degré de diversité génétique important.

Alors, si 10000 n'est pas un goulot responsable de la perte de la diversité génétique, quel est le phénomène en cause? Pour Jan Klein, un biologiste de l'évolution de l'Institut MaxPlank en Allemagne, ce phénomène pourrait être simplement dû à l'explosion de la population humaine survenue au cours des 10000 dernières années. Selon des simulations de la croissance des populations, la dispersion de reproduction qui accompagne une croissance rapide mélange les gènes à travers la population à un plus grand degré que dans une petite population qui ne se reproduit pas aussi fréquemment. Le résultat en est une tendance à l'homogénéisation génétique [1].

Nos ancêtres auraient survécu à un épisode où ils étaient en aussi grand danger que le sont aujourd'hui les chimpanzés pigmés ou les gorilles des montagnes. Cet épisode était vraisemblablement climatique, tel le refroidissement lors de la dernière période glacière du Pléistocène qui a pu être aggravé encore par la poussière émise lors de l'éruption du gigantesque volcan Toba à Sumatra il y a 73500 ans; le refroidissement a dû être de $5^{\circ} \mathrm{C}$ [2]. Un consensus semble exister sur les nombres mais les chercheurs pourront-ils un jour se mettre d'accord sur leur signification? L'étude de plus de gènes nucléaires chez l'homme et d'autres espèces de primates pourrait peut-être aider à mieux comprendre l'équilibre entre diversité et uniformité. En tout cas, à ce jour, les opinions sur le pourquoi nos gènes sont similaires restent très diverses.

E.D.

1. Gibbons A. The mystery of humanity's missing mutations. Science 1995 ; $267: 35-6$.

2. Takahata N, Satta Y, Klein J. Divergence time and population size in the lineage leading to modern humans. Theor Popul Biol 1995 (sous presse).

3. Gibbons A. Pleistocene population explosions. Science 1993 ; 262 : 27-8

4. Maynard Smith J. The Y of human relationship. Nature $1990 ; 344: 591-2$. 